\title{
Subjective Experience in Explanations of Animal PTSD Behavior
}

\author{
Published in Philosophical Topics: Ascriptions of Consciousness (Spring 2020)
}

\author{
Kate Nicole Hoffman \\ University of Pennsylvania \\ hoffmakn@sas.upenn.edu
}

\begin{abstract}
Post-Traumatic Stress Disorder (PTSD) is a mental health condition in which the experience of a traumatic event causes a series of psychiatric and behavioral symptoms such as hypervigilance, insomnia, irritability, aggression, constricted affect, and self-destructive behavior. This paper investigates two case studies to argue that the experience of PTSD is not restricted to humans alone; we have good epistemic reason to hold that some animals can experience genuine PTSD, given our current and best clinical understanding of the disorder in humans. I will use this evidence to argue for two claims. First, because the causal structure of PTSD plausibly requires reference to a traumatic conscious experience in order to explain subsequent behaviors, the fact that animals can have PTSD provides new evidence for animal consciousness. Second, the discovery of PTSD in animals puts pressure on accounts which hold that animal behavior can be fully explained without reference to subjective experience.
\end{abstract}

\section{ACKNOWLEDGMENTS}

Many thanks to Gay Bradshaw and the Kerulos Center, Lisa Miracchi and the MIRA group at the University of Pennsylvania, SUNY Potsdam's Presidential Scholars program, the audience at the 2019 meeting of the International Society for the History, Philosophy, and Social Studies of 
Biology, Michael Weisberg, and an anonymous reviewer for their feedback and help. Most of all, thank you to David Curry, who helped me think through countless early drafts of this paper.

In 1997, a chimpanzee called Jeannie was brought to the Fauna Foundation, a chimpanzee sanctuary located in Quebec, Canada. Jeannie had spent nine years at the New York Laboratory for Experimental Medicine and Surgery in Primates (LEMSIP) and was one of roughly 250 chimpanzees kept at the facility to be used for virology research. Although most of the chimps remained at the laboratory until it was closed in 1998 (where they were then transferred to another research facility), Jeannie was released in 1997 on account of demonstrating "serious emotional and behavioral problems" (Capaldo and Bradshaw 2011, 15). These behavioral problems included constant screaming, refusal to eat, pulling out hair and nails, and biting and hitting herself. As caregivers at the Fauna Sanctuary worked to rehabilitate Jeannie, they were able to learn about the conditions of her time spent at LEMSIP. Jeannie had been kept in a tiny cage suspended from the ceiling and had not been allowed to interact with any of the other chimpanzees. She had been used regularly for various invasive surgeries and was "knocked down" by a dart gun more than 200 times (Capaldo and Bradshaw 2011, 15). ${ }^{1}$ Thanks to the expertise and care of the staff at Fauna, Jeannie's symptoms improved while at the sanctuary. However, they never completely went away.

\footnotetext{
${ }^{1}$ It is worth noting that LEMSIP was considered one of most humane research labs; Suzanne Roy, program director of the advocacy group "In Defense of Animals," states that "LEMSIP stood alone among animal research labs for its open-door policy and responsiveness to humane concerns" (Revkin 1995).
} 
Jenny the elephant was only two or three years old when she witnessed the slaughter of the rest of her herd. ${ }^{2}$ She was captured and taken to the United States, where she was held in captivity by a man who kept her in chains, gave her very little social interaction, deprived her of food and water, and subjected her to frequent beatings. When Jenny was finally brought to the Dallas Zoo around the age of 10, the caretakers reported that she displayed strange behavioral patterns, including rocking, banging her head against the wall, lashing out suddenly at staff, and engaging in life-threatening self-injury. These symptoms were exacerbated after the death of one of the other elephants at the zoo with whom Jenny had spent a lot of time (Bradshaw 2009, 9598). Staff members at the Dallas Zoo believed that details of Jenny's past could explain current abnormal behavior.

In both of these cases, a certain kind of experience resulted in a significant change in behavior. Further, these behaviors bear a striking resemblance to a well-known human phenomenon: Post-Traumatic Stress Disorder (PTSD). In this paper, I will argue that the experience of PTSD is not restricted to humans alone. My aim will be to show that the discovery of PTSD in nonhuman animals (henceforth simply "animals") gives us important insight into the conscious animal mind, as well as puts pressure on accounts that hold that animal behavior can be fully explained without reference to subjective experience.

\section{FROM BEHAVIOR TO EXPERIENCE}

Behaviorism was once the prevalent way to understand both human and animal behavior. Behaviorists believed that all behavior can and should be described without reference to the

\footnotetext{
${ }^{2}$ It is unclear whether the killing was part of a routine cull, a capture, or an attack by poachers.
} 
mind. By replacing any talk of psychological events with concepts like "stimuli" and "conditioned response," the behaviorists endeavored to explain all behavior in terms of observable external stimuli, rather than internal mental processes and effects. The project sparked an upsurge of research, organized societies, and even sociopolitical worldviews (see Skinner 1948). Of course, the behaviorists did not need to deny that mental states exist in some form. Rather, as B. F. Skinner argued, "The objection to inner states is not that they do not exist, but that they are not relevant in a functional analysis" (Skinner 1953, 35). ${ }^{3}$

Although once a widely practiced research program, behaviorism has fallen out of favor among psychologists and philosophers. The cognitive revolution put renewed value on the causal power of the mind, and the project of explaining all behavior without reference to mental activity was recognized as, at times, downright impossible. ${ }^{4}$ Nevertheless, reference to the mental need not include or suppose any kind of phenomenal consciousness or subjective experience. Functionalism, a widely held perspective in theory of mind, implements a reductive strategy by characterizing mental states as being completely constituted by their causal (or functional) role. For example, on a functionalist picture, "pain" would be identified as the state which is often the result of an injury of some kind, and which tends to cause a predictable collection of beliefs (the belief that one is in pain), desires (the desire to stop being in that state), and behaviors (moving away from the painful stimulus, yelling, groaning, etc.) (Levin 2018). ${ }^{5}$ Pain plays an important causal role in the transition between a certain kind of input (the harmful stimuli), and a certain kind of output (pain-related behaviors). Any instantiation of this causal role, whether it be found

\footnotetext{
${ }^{3}$ For more on the foundations and motivations of behaviorist psychology, see Hull 1943; Skinner 1938; Thorndike 1905; Watson 1913.

${ }^{4}$ See for example: Chomsky 1959; Hempel 1966; Putnam 1963; Tolman 1948.

${ }^{5}$ See also Fodor 1968, and Putnam 1975, 325-41, 429-40.
} 
in a human, animal, or machine, is considered an instance of the mental state "pain." Notably, for the functionalist, the internal constitution of the mental state does not factor into its characterization. In other words, mental states like "belief," "pain," and even "fear" are defined and identified without reference to any subjective, felt experience.

One of my aims in this paper is to put pressure on this aspect of the functionalist picture, and any account which seeks to explain all behavior (in particular, all animal behavior) without specific reference to phenomenal consciousness. ${ }^{6}$ To do so, I will be examining certain kinds of behaviors from the perspective of mental health: in particular, the behaviors associated with Post-Traumatic Stress Disorder (PTSD). While phenomenal consciousness is typically excluded from functional descriptions of mental states, I will use current clinical models of PTSD's causal mechanism to argue that PTSD is unique in that its functional profile requires a conscious experience in the form of subjective horror or terror. In other words, PTSD's causal role cannot be specified without reference to subjective experience. Although this point certainly applies to human PTSD, I will be focusing on PTSD in animals. After demonstrating how documented behavior of abused chimpanzees and elephants fulfill the criteria for PTSD detailed in the Diagnostic and Statistical Manual of Mental Disorders V(DSM-V), I will argue that we have good reason to believe that PTSD can be experienced throughout the animal kingdom, albeit in varied ways. This is significant to the study of animal cognition since few animal behaviors require an explanation that makes explicit reference to subjective experience. I will argue that,

\footnotetext{
${ }^{6}$ Of course, there are many different types of functionalism, some of which might be more open to the inclusion of consciousness in understanding certain behavioral functions than others. For the sake of this paper, I will not be able to provide an analysis of these various formulations and how they might interact with my thesis about animal consciousness. Instead, I have provided a broad overview of the functionalist project, in order to demonstrate how a skeptic might agree that certain behaviors require mental explanations, but nevertheless believe that those mental explanations need not (and should not) make reference to subjective experience.
} 
since the functional profile of PTSD cannot be specified without invoking phenomenal consciousness, and since animals instantiate this profile, we have good epistemic reason to hold that these animals are having conscious experiences.

Typically, animal cognition is studied in the context of mental achievement. Instances of animal behavior that are considered indicative of consciousness tend to be examples of wellfunctioning cognitive behavior, often goal-oriented performances that are found to be too complex to describe purely in terms of the behavior itself. The behavior associated with PTSD, on the other hand, represents a kind of "malfunctioning" behavior that can nevertheless provide important insight into the conscious animal mind. The symptoms of PTSD are of particular importance if we are interested in studying animal awareness and experience for the purpose of ethics. PTSD symptoms constitute pain-related behaviors, and the traumatic experiences that serve as the origin of these symptoms overwhelmingly involve the interaction between humans and animals.

It is hard to imagine that the project of reducing mental states to their causal roles would in any way negatively affect the way that we view a human's capacity to have subjective experiences; after all, we can be pretty sure that all instances of human pain include a kind of unpleasant phenomenal experience, regardless of whether or not we think that the mental state should be characterized in terms of that experience. However, the same might not be true when it comes to animals. Given that there is a perceived difference between the way that we come to know that a human being is conscious and the way that we come to know that an animal is conscious, characterizing animal mental states without mention of phenomenal consciousness has the potential to cause harm. As I will argue more toward the end of the paper, distancing 
ourselves from the full acknowledgment of animal subjective experience could also serve to distance us from appreciating our ethical obligations to them.

The rest of the paper will be structured according to the following argument. First, I will use the DSM-V criteria in order to show that some animals plausibly exhibit PTSD according to our best clinical criteria and models. Second, I will demonstrate the ways in which these criteria and models make essential reference to conscious experiences in explaining both the causal role and the behavioral symptoms of PTSD. Finally, I will conclude that the best explanation for the behavior of animals with PTSD-like symptoms makes essential reference to their having conscious experiences, and that, therefore, we have very good epistemic reason to believe that these animals are conscious. For those who need no convincing that animals are conscious, the key takeaway will be that accounts which attempt to explain all animal behavior without any reference to subjective experience are hard pressed to explain PTSD, and this should affect whether or how we use these accounts when describing animal behavior (especially pain-related behavior). Along the way, I will draw out further implications of animal PTSD, including ethical implications, and answer some potential objections.

One final caveat. Although I believe that the evidence I am about to present makes a strong case for animal consciousness, my aim is not to convince the diehard skeptic. It is hard, if not impossible, to rule out the (in my mind, extremely distant) possibility that all or most cognitive states of human beings, including PTSD, could theoretically occur in animals without consciousness (see, for example, Carruthers 1998). Rather than attempting to conclusively rule out this possibility, my argument takes the form of an inference to the best explanation: given that the development of PTSD behaviors in humans seems to rely on conscious experience, and 
given that animals display an almost exact analogue of these behaviors and can be treated via similar means, the best explanation is that these animals are experiencing a form of PTSD that relies on conscious experience. ${ }^{7}$ Again, my project is not to convince the most stubborn of skeptics, but rather to contribute to this field a new and ethically relevant piece of evidence for animal consciousness.

\section{EVIDENCE OF ANIMAL PTSD}

Let's return to Jeannie's story. Unsurprisingly, the behaviors displayed by Jeannie after her time at LEMSIP are extremely abnormal for a chimpanzee and are almost never seen in the wild (Ferdowsian et al. 2011, 8). It seemed clear to the caretakers at the Fauna Sanctuary that Jeannie's behaviors were the result of extreme, human-induced distress. In particular, Jeannie's symptoms seemed to closely resemble a rather sophisticated kind of pain-related behavior seen in humans: Post-Traumatic Stress Disorder, where an extraordinary event causes a significant change in behavior. In fact, caretakers at the Fauna Sanctuary developed their rehabilitation strategies and caregiving according to the assumption that Jeannie was, in fact, suffering from PTSD. The same was true of the staff at the Dallas Zoo, as they sought to understand the abnormal behavior of Jenny the elephant. Renowned conservationist Dame Daphne Sheldrick, who rescued and successfully rehabilitated hundreds of orphaned elephants like Jenny, believed that abnormal elephant behavior could often be explained in terms of something like PTSD, writing, "Over the years we have learned a lot about the disturbed psychological state of traumatized orphaned elephant young. Their behavior often mirrors the post-traumatic stress

\footnotetext{
${ }^{7}$ See Jonathan Birch's "theory-light" approach to thinking about the connection between consciousness and cognition (Birch 2020).
} 
syndrome of humans” (Sheldrick 2012, 308). Ecologist/psychologist Gay Bradshaw has developed a new field of research (called trans-species psychology) with the intention of demonstrating that animals like Jeannie and Jenny can and should be diagnosed with PTSD. ${ }^{89}$ However, these kinds of diagnoses are rare, and tricky. Even though it is not unusual to hear someone refer to an animal's behavior as indicating PTSD, the behaviors cited usually go no further than an abused dog shrinking away at the sight of a human, or their extreme startle response to certain noises. ${ }^{10}$ Rarely is a case of supposed animal PTSD truly considered the kind of cognitively and emotionally rich experience that we ascribe to a human suffering from a mental disorder.

Although it would be easy to say that the reason animals are not typically diagnosed with mental disorders is simply because we tend to underestimate the sophistication of animal minds, there are some real and serious practical problems. Chief among them is the fact that some psychological symptoms required for a diagnosis seem to only be made known through selfreport. Let's take a look at the criteria that a human must meet in order to be diagnosed with PTSD according to the Diagnostic and Statistical Manual of Mental Disorders $V$, which is used in current practice to diagnose and prescribe. According to the DSM-V, a human can be diagnosed with PTSD if they have had exposure to a traumatic event and experience symptoms from the four symptom clusters: intrusion symptoms (which involve the inability to keep out thoughts of the trauma); persistent effortful avoidance of distressing trauma-related stimuli (the

\footnotetext{
${ }^{8}$ Jeannie the chimp and Jenny the elephant are explicitly discussed in Bradshaw 2009 and Capaldo and Bradshaw 2011.

${ }^{9}$ Trans-species psychology is meant to reflect the corpus of science, and integrates neuroscience, psychology, and ethology to explain and understand animal minds.

${ }^{10}$ Not that I want to necessarily rule out these behaviors as being evidence of PTSD in dogs (more on this later).
} 
tendency and desire to avoid reminders of the trauma); negative cognitions/mood (deterioration of mood); and alterations in arousal and reactivity (change in levels of arousal, either hyperaroused or hypo-aroused). More specific criteria for the diagnosis of human PTSD are as follows: ${ }^{11}$

In order for a scenario to be considered a trigger for PTSD, the individual must have either:

1. Directly experienced the traumatic event

2. Witnessed the traumatic event in a person

3. Learned that the traumatic event occurred to a close family member or friend

4. Experienced first-hand repeated or extreme exposure to aversive details of the traumatic event

Intrusion Symptoms (must have at least one symptom for diagnosis): Recurrent, intrusive memories; traumatic nightmares; flashbacks; intense/prolonged distress after exposure; physiologic reactivity upon exposure to cues

Persistent, effortful avoidance of distressing trauma-related stimuli (must have at least one symptom for diagnosis): trauma-related thoughts/feelings; trauma-related external reminders

Negative cognitions/mood (must have at least two symptoms for diagnosis not present before traumatic event)

Inability to recall key features of the trauma; negative beliefs or expectations about oneself/others/the world; distorted blame of self/others; persistent negative trauma-related emotions; diminished interest or participation in significant activities; feeling alienated; constricted affect

Alterations in arousal and reactivity (must have at least two symptoms for diagnosis not present before traumatic event): Irritable or aggressive behavior; selfdestructive/reckless behavior; hypervigilance; exaggerated startle response; problems in concentration; sleep disturbance

Additional criteria: symptoms must persist for over a month, and cause "clinically significant distress."

\footnotetext{
${ }^{11}$ For more information, see the DSM-V 271-80.
} 
As is evident, the current criteria for human PTSD are fairly complex, and, in some cases, vague. Diagnosing animals faces an additional problem. Although a human might be able to tell us that they are experiencing recurrent, intrusive nightmares, flashbacks, or trauma-related thoughts or feelings, we do not have the same luxury with a chimpanzee or elephant. ${ }^{12}$ As physician and advocate Hope Ferdowsian puts it:

Efforts to estimate the prevalence of psychiatric disorders in chimpanzees and other animals encounter special challenges. Diagnostic criteria for mood and anxiety disorders typically require verbal descriptions from subjects of their experiences and internal states. The inability of nonhuman primates to report symptoms presents obstacles similar to those in pediatrics, psychiatry, and geriatrics, often requiring special investigative methods ... (Ferdowsian et al. 2011, 2)

Given this challenge, many of the self-report symptoms that could count toward a human PTSD diagnosis cannot be counted toward an animal diagnosis. One possible solution is to simply change the criteria required in order for an animal to be diagnosed with PTSD. ${ }^{13}$ However, for the sake of this paper, I want to focus on comparing Jeannie and Jenny's behavior with the standards set out in the DSM-V without alteration. My aim is to show that animals can experience PTSD in the first place, and I worry that such alterations leave room for a critic to claim that the fact that the standards were changed or lowered gives us reason to believe that animals don’t experience "real" PTSD (or at least, since I am mainly interested in the epistemic point, that "real" PTSD is not the best explanation for the animals' behavior). Although this

\footnotetext{
${ }^{12}$ Although there are serious limitations to self-report even in humans, and it is widely recognized that it would be naïve to accept all (or even most) cases of self-report as reliable (Stone et al. 2009). In some cases, observations of behavior, and not self-report, will be the best way to diagnose humans with mental disorders.

${ }^{13}$ Ferdowsian has developed a set of alternative criteria for diagnosing animals with PTSD, which removes or alters those DSM-IV criteria based on self-report, changing the standards that an animal must meet in order to be diagnosed (Ferdowsian 2011). If diagnosing animals with mental disorders becomes more standard, this kind of alternative criteria will almost certainly be more successful in determining which animals should be diagnosed.
} 
means that some symptoms, those which are difficult or impossible to detect from the outside, cannot be used in order for an animal to qualify for a diagnosis, we will nevertheless keep the standards exactly the same. ${ }^{14}$ In other words, we are trying to determine whether, even with a reduced number of potential symptoms, Jeannie and Jenny still have the required amount and kind of symptoms necessary to be diagnosed with PTSD, according to the DSM-V.

Jeannie was observed by members of LEMSIP and the Fauna Foundation, and reportedly displayed the following behavioral symptoms (Capaldo and Bradshaw 2011):

\section{Intrusion symptoms (requires one symptom):}

1. Physiologic reactivity upon exposure to cues $^{15}$ : Jeannie would have frequent episodes in which her eyes would "roll about" and she would appear to be completely unaware of her surroundings. She would not "come back" from these episodes without external intervention. ${ }^{16}$

\section{Persistent, effortful avoidance of distressing trauma-related cues (requires one symptom):}

1. Trauma-related external reminders: inability to tolerate touch from either humans or other chimpanzees (refused grooming), displayed irritable behavior when anyone approached her enclosure

\section{Negative cognitions/mood (requires two symptoms):}

\footnotetext{
${ }^{14}$ Even if that means that, among those symptoms which can still be found to be applicable to animals, the behavioral aspects will be emphasized. This strategy is not unique to diagnosing animals; nonverbal children and adults who are unable to self-report can nevertheless be diagnosed with PTSD, as certain behaviors are taken to be indicative of the fulfillment of DSM-V criteria. More on this later.

${ }^{15}$ More typical physiological signs of PTSD would include increased heart rate, blood pressure, and temperature, sweating, nausea, increased cortisol levels, muscle tension, etc. Because neither Jeannie nor Jenny were tested for these symptoms, I have not included them here. However, I will note that animals have been shown to display the brain and blood markers associated with PTSD after being exposed to a traumatic event (for example, see Cooke et al. 2017, which details these physical symptoms in cows after a staged wolf encounter).

${ }^{16}$ Although one could argue that this symptom is more indicative of a medical condition than a psychological disorder, according to the staff at LEMSIP and the Fauna Foundation the "seizure-like episodes ... fit no known physiological pattern consistent with neurological damage" (Bradshaw et al. 2008, 15). Furthermore, the fact that this symptom improved over time at sanctuary suggests that it was not merely a medical condition.
} 
1. Negative beliefs or expectations about oneself/others/the world: (although we can make no claim about "beliefs," these behaviors do seem to indicate a reduced level of trust and safety) - hesitant and fearful in new environments, avoided eye contact, guarded 2. Diminished interest or participation in significant activities: not motivated to learn new skills, often found in a near vegetative state

3. Feeling alienated: withdrawn, limited social interaction and skills

\section{Alterations in arousal and reactivity (requires two symptoms):}

1. Irritable or aggressive behavior: engages in "ritualistic eating behaviors" and "ritualistic circling," attacks cage mates and human caretakers

2. Self-destructive/reckless behavior: biting and hitting self, pulls off hair and nails

3. Hypervigilance: hypervigilant and hyperaroused - very difficult to calm, awoke startled, reacts to minor changes in lighting

4. Exaggerated startle response: extreme responses to minor stress

Because Jeannie's symptoms manifested only after her time at LEMSIP, match the required kind of symptoms in terms of symptom clusters, match the required amount of symptoms required per symptom cluster, and persisted for over a month, Jeannie could, according to the DSM-V standards originally created for humans, be diagnosed with PTSD.

Jenny the elephant's behaviors were documented by staff at the Dallas Zoo, and compiled in Bradshaw's book Elephants on the Edge (2009):

\section{Intrusion symptoms (requires one symptom):}

1. Intense/prolonged distress after exposure: Jenny showed "extreme distress" (especially after death of companion), including head banging, screaming, and running through her room.

2. Physiologic reactivity upon exposure to cues: any kind of confinement brings about stereotypic behaviors including rocking, banging, and kicking feet against the wall, spinning in circles, screaming, shaking, pressing her head against the ground, and repeatedly swinging her leg back and forth.

\section{Persistent, effortful avoidance of distressing trauma-related stimuli (requires one symptom):}


1. Trauma-related external reminders: Jenny reacted negatively to confinement (see above) and the presence of humans: exhibited extreme aggression toward zoo caretakers and health-care workers.

\section{Negative cognitions/mood (requires two symptoms):}

1. Diminished interest or participation in significant activities: refusal to eat, engage in social activity, or play

2. Feeling alienated: sits motionless for hours at a time, does not engage in nonaggressive social interaction.

2. Constricted affect: reported by staff members as being "moderately depressed"17

\section{Alterations in arousal and reactivity (requires two symptoms):}

1. Irritable or aggressive behavior: sudden attacks against staff members and other resident elephants (lashing out, chasing down, shoving against walls). Aggression so extreme that Jenny had to be physically restrained.

2. Exaggerated startle response: demonstrates "low resistance to stress"

3. Self-destructive/reckless behavior: strikes right leg repeatedly, resulting in chronic injuries and difficulty standing.

Like Jeannie, Jenny's behaviors match up with the DSM-V's PTSD criteria. In fact, Jenny's case study was sent out to five mental health professionals, who reviewed her history and subsequent behaviors. Jenny was referred to only as a thirty-two-year-old female called "E.M.," and the fact that she was an elephant, and not a human, was not revealed. All five professionals diagnosed “E.M.” with PTSD (Bradshaw 2009, 108).

Before continuing, I want to respond to a possible objection. Although I have claimed to be focusing solely on behavioral reports in this section, some of these criteria, especially those in

\footnotetext{
${ }^{17}$ Although we don't want to make any claims about depression yet, the fact that the staff members reported Jenny as being depressed indicates that she displayed certain kinds of behaviors which are often shown by depressed humans. Since these behaviors would be taken to fulfill this criterion in the human case, the same can be said in this case.
} 
the "negative moods/cognitions" symptom cluster, appear to require assumptions about the animals' mental states. Since I am using the fulfillment of this criteria in order to show that we should be referring to mental states in order to explain the abnormal behavior, the argument might appear circular. However, even in the human case, certain behaviors are taken to be measures of relevant beliefs and feelings, even when those beliefs and feelings are not explicitly reported. This is especially true when the traumatized person is nonverbal, or unable to properly express their thoughts and emotions in verbal form. The criteria for a PTSD diagnosis in young children (DSM-V, 272-74) recognizes the distinct importance of behavioral markers when it comes to diagnosing children six years and under. For example, whereas a criterion in the "negative moods/cognitions" symptom cluster for adults/older children requires that the patient have a "persistent inability to experience positive emotions" (272), the DSM-V lists the corresponding criterion for young children as the "persistent reduction in expression of positive emotions" (273). The criterion for flashbacks in young children is met when a child "feels or acts as if the traumatic event(s) were recurring" (273). Whereas in adults/older children, PTSD's impact on sociality is determined in terms of "feelings of detachment or estrangement from others" (272), in young children it is marked by "socially withdrawn behavior" (273). The DSMV notes that "Because of young children's limitations in expressing thoughts or labeling emotions, negative alterations in mood or cognition tend to involve primarily mood changes" (277). In all of these cases, certain behavioral patterns are recognized as legitimate measures of the relevant DSM criteria.

No doubt a skeptic will argue that, still, there is enough of a difference between a six-yearold human and an animal for the analogy to break down. However, to see just how strong the 
parallels are between the methodology I am using here and the methodology used when diagnosing nonverbal children, we will briefly turn to Scheeringa et al.'s (1995) work on applying diagnostic criteria to traumatized infants under the age of two. ${ }^{18}$ Scheeringa et al.’s task is to determine whether or not infants newborn to age two can develop PTSD, despite their limited cognitive and emotional capacities, and, if so, the degree to which the DSM can successfully diagnose. The parallels should already be obvious: for one, as in the case of animals, one might doubt at the outset that infants so young could experience something as complex as PTSD. Additionally, Scheeringa et al. recognize that the biggest challenge in diagnosing infants is their inability to verbally report symptoms. ${ }^{19}$ To determine whether infants can nevertheless accurately be said to have the capacity for PTSD, the team collected and studied published case studies in which infants experienced a traumatic event and displayed marked behavioral patterns afterward. Although the infants had many of the symptoms commonly taken to be indicative of PTSD, and although these symptoms only manifested after a traumatic encounter, none of them qualified for a diagnosis according to the standards set out in the DSM (Scheeringa et al. 1995, 194). Here is the first major difference between human infants and animals like Jeannie and Jenny: only in the animal cases do behavioral patterns fully meet the DSM criteria for a PTSD diagnosis.

\footnotetext{
${ }^{18}$ See also Drell et al. (1993) for more on PTSD in children ages 0-3, and how developmental considerations in children so young might affect the phenomenology of the experience.

${ }^{19}$ Scheeringa writes: "A formidable challenge in evaluating PTSD in infants is their limited ability to convey their subjective experiences. Their limited cognitive and expressive language skills make inferring their thoughts and feelings difficult. Current diagnostic criteria in the DSM-IV, for example, contain eight items that require verbal descriptions from patients of their experiences and internal states. This leaves only 10 of 19 items that can be rated in infants with confidence" (Scheeringa et al. 1995, 191).
} 
Notably, even though the infants did not qualify for a PTSD diagnosis under the DSM, Scheeringa et al. did not thereby conclude that infants cannot experience PTSD. ${ }^{20}$ Instead, they argue that the more plausible possibility is that the DSM criteria, having been developed to diagnosis individuals capable of verbal report, cannot accurately detect cases of PTSD in nonverbal infants. For them, the solution is simple: alter the DSM criteria to allow for diagnoses based on behavior. They write:

One of the major changes incorporated into the new criteria was to make the items more objective and anchored in observable behaviors. Subjective experiences, that is, thoughts and feelings, are impossible or very difficult to value reliably in preverbal or barely verbal children ... The alternative criteria, which attempt to reduce the need to make inferences about thoughts and feelings, had better interrater reliability than the DSM-IV criteria. $(199)^{21}$

Using these altered criteria, the majority of the infants in the case studies could be diagnosed with PTSD. Scheeringa and his team took this to indicate the superior validity of the alternative criteria as applied to infants. $^{22}$

There are a few points that I want to pull out of this discussion. First, it is clear that mental health professionals are perfectly happy to allow certain behavioral patterns to count toward a PTSD diagnosis when verbal report of subjective thoughts or feelings is impossible. Still, a critic

\footnotetext{
20 "The second hypothesis, that children younger than 48 months of age might not manifest enough persistent signs and symptoms to develop a posttraumatic syndrome after a severe trauma, also was not supported. Almost all of the infants in the total 32 cases showed multiple signs that were recognized as impairments from their usual level of functioning and lasted longer than 1 month. Furthermore, all were referred to mental health professionals for treatments" (Scheeringa et al. 1995, 198).

${ }^{21}$ PTSD criteria that rely more on behavioral markers than verbal report might also be beneficial in diagnosing some patients on the Autism spectrum (see Mehtar and Mukaddes 2011 and King 2010).

${ }^{22}$ Recall that Ferdowsian 2011 uses Scheeringa's alternative criteria to diagnose chimpanzees with PTSD. She too believes that the alternative criteria is more accurate in identifying cases of PTSD among chimpanzees, given its reliance on behavioral markers rather than verbal report.
} 
might say, I am supposing too much. In the infant case, we can be pretty sure that these behavioral patterns really are indicative of underlying thoughts and emotions. In the animal case, this is the very question under discussion. Although I may not be able to convince the most stubborn of animal consciousness critics, I believe that we have very good reason to find the analogy between nonverbal infants and animals informative. One might worry that animals simply do not have the cognitive or emotional capacities to truly experience PTSD, whatever their behavioral patterns might seem to indicate. But infants too have limited cognitive and emotional capacities (see Scheeringa et al. 1995, 192, for a list of infant abilities taken to be relevant to PTSD). Yet despite these limitations, and despite the fact that infants do not even qualify for a PTSD diagnosis under normal DSM criteria, mental health professionals still feel confident that infants can experience PTSD (so confident, in fact, that they are willing to adjust the DSM criteria to better reflect this). ${ }^{23}$ Given that chimpanzees and elephants are often considered to have intelligence levels higher than that of a two-year-old human (in relevant domains like memory: see Byrne et al. 2009; Inoue and Matsuzawa 2007) and given that these animals do qualify for a PTSD diagnosis under the original DSM criteria, any claim that PTSD can occur in human infants, but not in animals, must be based on some kind of principled divide between human and animal minds. If we are not content to assume this divide, and are instead sensitive to the empirical evidence, then we ought to think that, if nonverbal infants and children can legitimately be diagnosed with PTSD, the same should be true of animals like Jeannie and Jenny.

\footnotetext{
${ }^{23}$ See also: Blank 2007; Bogat et al. 2006; Feldman and Vengrober 2011; Graf et al. 2011; Scheeringa et al. 2001.
} 


\section{PTSD AND CONSCIOUS EXPERIENCE}

According to a reasonable extrapolation from the standards set out by the DSM-V, humans are not the only creatures who can be diagnosed with PTSD. But what does this tell us about animal subjective experience? Although both human and animal behaviors can be matched up with PTSD criteria, perhaps only the human form of PTSD is complex enough to require an explanation that includes various mental states or conscious experiences. The symptoms displayed by animals, although unusual, might be mere automatic, unconscious bodily responses to negative stimuli (as a skeptic with behaviorist leanings might say; for example, see Kennedy 1992). In other words, although certain animal behaviors might resemble PTSD, one might argue that it would be an error to seriously relate these symptoms to real, human PTSD, or to think that these behaviors are in any way indicative of animal consciousness.

However, there is good reason to think that these animal behaviors are not mere bodily responses. First, while we might be able to come up with "simple" stimulus-reaction explanations for symptoms like exaggerated startle response or reaction to trauma-related external reminders, it seems less plausible for self-harm, diminished interest, and refusal to eat, especially when these behaviors occur absent any potential negative stimuli. These more complex behaviors resist simple stimulus explanations and demand a kind of explanation that goes beyond mere description of behavior to include some reference to the mind (of course, whether this reference to the mind needs to include phenomenal consciousness is a further question, which I will get to momentarily).

Second, there is evidence that PTSD symptoms in animals can be successfully treated using methods usually offered to human patients. Since PTSD treatments in humans do not only 
address negative associations to stimuli (although some do, e.g., exposure to sounds or sights that cause reactions in order to "re-train" the brain), this implies that the parallels in symptoms extend beyond pain reaction. For example, the experience of a traumatic event often leads to feelings of powerlessness, and then, as a result, further hopelessness and depression. Therapists attempt to address this effect of PTSD by working to make sure that the patient feels that they are safe, secure, and in control. They might do this by pointing out the ways in which the individual does have control over her life, and perhaps even providing opportunities for the patient to exercise this control. With this same goal in mind, sanctuary workers at the Fauna Foundation strive to "restore and reinforce the chimpanzee's sense of agency and safety" (Bradshaw et al. 2008, 24). Chimps are given opportunities to choose what they want to eat, with whom they socialize, when they sleep, and what they play with: choices that they were denied in their former lives in research facilities. In both human and chimp cases, regaining a sense of agency through the ability to make choices has been shown to be crucial in reducing PTSD symptoms (Bradshaw et al. 2008, 24). Additional treatments offered to both humans and animals include therapeutic socialization and medication (Bradshaw et al. 2008, 15, 29). ${ }^{24}$ The fact that these treatments have been shown to work provides evidence that these animals' condition is functionally similar to that of humans with PTSD. At the very least, it makes it clear that the symptoms are far more complicated than an automatic and purely bodily response to harmful stimuli. This makes it hard to see how the animals' behavior could be explained using concepts like conditioned response or negative reinforcement alone.

\footnotetext{
${ }^{24}$ For example, Jenny was prescribed Phenobarbital and Acepromazine; these medications were found to be somewhat successful in reducing symptoms (Bradshaw 2009, 96).
} 
Of course, even if we grant that the simplest, best explanation for PTSD behavior involves the mind, explanations in terms of the mental need not involve subjective experience; one might refer to the causal role of mental states without invoking facts about the phenomenology of the mental states themselves. However, PTSD resists this kind of reductive strategy since the functional role of PTSD cannot be adequately characterized without subjective experience. The clearest way to see this is to reflect on the nature of the disorder itself. The behavior demonstrated by Jeannie and Jenny occurred only after a certain kind of event took place; something about their experience changed the way that they behaved in the long term. Psychologists prefer to think of the occurrence of PTSD in terms of a collection of risk and protective factors: risk factors increase the chances of an experience of PTSD, and protective factors decrease the chances. For humans, risk factors include membership in disadvantaged groups ${ }^{25}$ preexisting psychiatric conditions, and potentially even genetic factors (Ford et al. 2015). Although these factors and others contribute to whether or not a person will develop PTSD after experiencing a traumatic event, no one factor guarantees it.

Yet above all of these various risk and preventative factors stands the most important factor of all in determining the chances that a person will develop PTSD: the traumatic event itself. The more traumatic an event is, the more likely a person is to develop PTSD, regardless of the other contributing risk or preventative factors. Psychologists attempt to measure this factor in terms of the "degree or severity of exposure" to the traumatic event (Ford et al. 2015, 93), which ultimately determines the likelihood that the event will cause trauma. Of course, determining whether or not a certain event should be considered traumatic, and, if so, how traumatic it is, is a

\footnotetext{
${ }^{25}$ This is because members of disadvantaged groups are more likely to experience certain kinds of traumatic stressors and are also more likely to lack the social and political support that could aid in resilience.
} 
tricky affair, and has been hotly debated. One extremely plausible position is to simply hold that a traumatic event can only be measured by 'the survivor's subjective response of terror or horror" (Ford et al. 2015, 93). In other words, any judgment about whether, or the degree to which, an event was traumatic can only be informed by an understanding of the subjective state of the individual who experienced the event. If we are to take seriously this account of trauma, then talk of subjective experiences cannot be divorced from any discussion of PTSD, human or otherwise. Usually, the causal role of a mental process can be specified without reference to any phenomenal experience (recall how a functionalist might define belief, pain, or fear). But if traumatic events can only cause a change in behavior through subjective experiences of terror, then PTSD's functional role, the way in which it facilitates the transition between a certain input (a traumatic event) and an output (PTSD behaviors) cannot be properly described without consciousness. In other words, if we are truly interested in characterizing and explaining PTSD, including how a traumatic event can be causally related to a specific set of behaviors, our explanation will need to include information about the phenomenology of a certain kind of mental state.

The same might be said of the PTSD symptoms themselves. Both humans and animals can be diagnosed with PTSD based on exhibited behavioral patterns alone. However, when we move beyond using various behaviors as measures for the fulfillment of DSM criteria, and look to characterizing the symptoms themselves, it seems clear that a richer and more plausible description of those symptoms will need to include subjective, conscious experience. It is hard to characterize symptoms like "diminished interest" in terms of behavior alone: we understand what it $i s$ to have diminished interest (or nightmares, alienation, constricted affect, etc.) in terms of 
consciousness. The best way to explain and describe the symptoms that result from the experience of a traumatic event involves not only a description of behaviors, but also the various phenomenological experiences associated with those behaviors. ${ }^{26}$

What does this mean for discussions about animal consciousness? As I've said, it is rarely the case that any notion of subjective experience must be referenced in describing or explaining animal behavior. Although a researcher need not deny that a certain behavior may (or even may very likely) include a subjective experience, typically accompanying psychological descriptions are considered, at best, unnecessary, or, at worst, a scientific overstep. However, given what we know about the role of subjective terror in the manifestation of PTSD behaviors, we here have a kind of animal behavior that cannot be adequately described or explained without reference to conscious experience.

\section{LIMITATIONS AND OBJECTIONS}

I have argued that we have good epistemic reason to hold that some animals can experience genuine PTSD, given our current and best clinical understanding of the disorder in humans. Because the causal structure of PTSD plausibly requires reference to a traumatic conscious

\footnotetext{
${ }^{26}$ Note that it is not necessary for the animal to have a conscious memory of the traumatic event in order for them to experience symptoms. Ford et al. explain that “... PTSD can occur despite the individual having no conscious memory of traumatic events. This is especially relevant for people who were exposed to maltreatment or family or community violence in the first years of life and subsequently develop PTSD symptoms" (Ford et al. 2015). [AU: There is no Ford et al. 2015 in the references; there is a 2009. Please advise.] The subjective terror experienced in a traumatic event can leave an imprint on one's mind which could result in symptoms even when an individual might be unable to report or even remember their experience (even though, at the time of occurrence, the experience was conscious). Despite this lack of memory, the symptoms themselves would still be consciously experienced (in both human and animal cases, as I have argued). This point might help to moderate worries that animal memory is not sophisticated enough to allow for a persistent conscious memory of a trauma. The ability to remember the traumatic event, although perhaps relevant to the overall experience of PTSD, does not ultimately determine whether or not PTSD symptoms can develop.
} 
experience in order to explain subsequent behaviors, the fact that animals can have PTSD provides new evidence for animal consciousness. I will now turn to some limitations and possible objections.

Maybe we agree that chimpanzees and elephants can experience PTSD. But what about bears? Birds? Bees? What about our pets? Do we need to make sure that their behavior fits the DSM-V criteria in order to conclude that they too can experience PTSD? If they don't fully qualify for PTSD under these criteria, does that mean that they are not suffering as a result of a conscious traumatic experience? What is the use of a mental health perspective if it is limited to a couple of higher mammals?

These questions bring up two important points. The first is that PTSD is not a uniform experience, even among humans. The kinds of symptoms that arise from a traumatic encounter, the way in which those symptoms are experienced, and the kinds of interventions that will aid in reducing symptoms often vary according to a person's age, gender, race, culture, and/or social condition (Ford et al. 2009; Wilson 2007). If PTSD can be so varied even among humans, then there is very good reason to believe that it will vary among animals as well (both among individuals, and from species to species). If this is the case, then the DSM-V criteria may not be flexible enough to detect cases of PTSD widely across species.

There is a lot of philosophical and psychological literature that aims to understand the nature of mental disorders, and a fair amount of that literature criticizes the effectiveness of the DSM in providing definitions of mental disorders and criteria for their diagnoses. ${ }^{27}$ In the philosophy of depression literature, for example, Matthew Ratcliffe argues that depression

\footnotetext{
${ }^{27}$ For a small sample, see Bueter 2019; Cooper 2006; Lalumera 2016; Tekin and Mosko 2015; Tsou 2016.
} 
should be primarily understood not as a set of behaviors, but rather as a fundamentally phenomenological experience, which is constituted by a change in the way that one finds themselves in the world (Ratcliffe 2015). According to Ratcliffe, the disorder should be defined in terms of a loss of various phenomenological possibilities as experienced by the depressed person. ${ }^{28}$ There are many other possible definitions of depression (or obsessive-compulsive disorder, or PTSD, or any other mental disorder) that one might think does better justice to these experiences than the DSM-V. Furthermore, these kinds of definitions might better accommodate varieties of experience, which nevertheless might all plausibly fall under the same category of disorder.

With these worries in mind, it might appear that my reliance upon the DSM in this paper is a mistake. I certainly do not want to commit myself to the claim that the DSM criteria, or behaviorally based definitions of mental disorders in general, most accurately captures the experience of PTSD or any other mental disorder (as I hope my discussion of the necessity of consciousness in fully characterizing PTSD symptoms has shown). Nevertheless, it seems clear that the DSM has been generally successful in helping practitioners to identify cases of mental disorders, which is why the DSM-V is used in practice to diagnose and prescribe today. ${ }^{29}$ Even if the DSM by no means perfectly characterizes PTSD, and even if it could or should be adjusted in order to better accommodate more varied experiences, it still cannot be denied that, in general, the criteria listed in the DSM-V is often very good at correctly identifying cases of PTSD. My

\footnotetext{
${ }^{28}$ Although Ratcliffe himself acknowledges that these changes are extremely difficult for a person with depression to report: "In fact, many autobiographical accounts of depression include the claim that the experience or some aspect of it is indescribable" (Ratcliffe 2013, 1).

${ }^{29}$ The American Psychiatric Association reports that the DSM-V is "the product of more than 10 years of effort by hundreds of international experts in all aspects of mental health" (American Psychiatric Association website).
} 
intention in using the DSM-V in my argument was merely to show that, since the DSM-V is generally successful in picking out cases of human PTSD, we have good reason to believe that it can successfully pick out cases of animal PTSD as well.

Given this, what are we to say about animals who may seem to display PTSD-like symptoms, but do not qualify for PTSD according to the DSM-V? It is important to show that some animals completely meet the DSM-V criteria for PTSD in order to demonstrate that it is not a mere anthropomorphic blunder to associate certain animal behaviors with what we typically take to be a complex human experience. But once we can appreciate that PTSD experiences are not restricted to humans alone, the importance of the DSM drops out. An abused dog exhibiting a heightened startle response and withdrawn behavior may not qualify for a strict PTSD diagnosis, but, given that we know that animals can experience PTSD, and that PTSD experiences can vary, it seems reasonable to say that the dog's behavior can be explained with reference to a consciously experienced trauma. Recognizing the continuum of psychological symptoms that can result from an encounter with a traumatic event pushes us to understand and describe certain pain-related animal behaviors in terms of not just stimulus reactions or even functional mental processes, but subjective, conscious experiences.

\section{CONCLUDING THOUGHTS: FURTHER IMPLICATIONS}

By analyzing certain animal behaviors through the lens of mental health, I have argued that PTSD is not restricted to humans alone, and that it provides us with a set of animal behaviors that can only be fully explained and appreciated with reference to subjective experiences. To conclude, I will briefly describe the ways in which the perspective of mental health might lead to 
further questions and insights when it comes to understanding animal minds. First, we might wonder what PTSD says about the cognitive abilities of the animals who can experience it. Scientists are currently studying the cognitive processing capacities which play a role in the development of PTSD after an experienced traumatic event (for example, see Brewin 2001; Dalgleish 2004). If PTSD is ultimately the result of a certain kind of mental process (which may be bound up in information processing, memory, etc.), then we might be able to gain new insight into the mental capacities of certain animals without ever having to bring them to a lab. If we are interested in the emotional capacities of animals, including their range of feeling or the importance of social connections, then we need look no further than the kinds of events that consistently cause trauma (the killing of one's family members, forced isolation, etc.).

There are further questions to be asked. For one, how many other species experience PTSD symptoms? Although this is a relatively new area of study, there is evidence of potential PTSD in various bird species, as well as dogs, cats, cows, and maybe even rats. ${ }^{30}$ How does the experience of PTSD change depending on the capacities of the different species? Can animals have any other kind of mental disorder? What kinds of situations are likely to cause trauma in animals?

This final question leads us directly into the most obvious implication of animal PTSD: our ethical obligations toward animals. Most people accept that animals can suffer. But talk of animal suffering is usually about physical suffering, not mental suffering. And even then, it is often assumed that, although animals may suffer, such suffering cannot truly be compared with human suffering. The fact that animals can have PTSD puts pressure on the idea that animal

\footnotetext{
${ }^{30}$ Bradshaw et al. 2009; Cooke et al. 2017; Dodman 2016; Nagasawa et al. 2012; Serova et al. 2013; Siebert 2016; Vermeire et al. 2009; Zanette et al. 2019.
} 
suffering is inherently "lesser" or even "simpler." But there are subtler ethical implications as well. A central aim of this paper has been to argue that we have good epistemic reason to hold that at least some animals are capable of having conscious experiences since, as a matter of practice, PTSD cannot properly be described without invoking consciousness. To those who find skepticism about animal consciousness implausible, this point might seem silly. Evolutionary theory predicts continuity across the animal kingdom, which would make the presence of consciousness in humans, and humans alone, nothing short of miraculous. Most pet owners are likely to find arguments that suppose that animals might not be conscious to be absurd. Nevertheless, at least in practice, explanations of animal behavior that make no reference to anything "in the head" (or, if they do, that make no reference to phenomenal consciousness) are considered more scientifically tractable, since these explanations are thought to be more likely to avoid the dreaded anthropomorphism that could come as a result of assuming certain kinds of animal experiences. ${ }^{31}$ But when we don't fully acknowledge the extent of animal awareness, we can feel justified in turning a blind eye to some of the ways that we continue to mistreat them. The way that we talk about animals, including how we explain their behavior, makes a difference to how we think about them. By shying away from explanations of animal behavior that make explicit reference to subjective experiences, we risk sidelining the importance of animal awareness. In thinking more carefully about the kinds of explanations that we should use when attempting to understand animal behavior, we may change not only the way that we think about

\footnotetext{
${ }^{31}$ Of course, a researcher's willingness to attribute certain kind of conscious states to animals often depends on the kind of animal being studied; it might seem cogent to label a chimpanzee's behavior as indicating consciousness, but not a bee's.
} 
the minds of other creatures, but also about how we as humans can begin to fix our broken relationship with them.

\section{REFERENCES}

Birch, Jonathan. 2020. "The Search for Invertebrate Consciousness" [Preprint].

Blank, Myles. 2007. "Posttraumatic Stress Disorder in Infants, Toddlers, and Preschoolers." British Columbia Medical Journal 49(3): 133-38.

Bogat, Anne G., et al. 2006. “Trauma Symptoms among Infants Exposed to Intimate Partner Violence." Child Abuse and Neglect 30(2): 109-25.

Bradshaw, G. A. 2009. Elephants on the Edge: What Animals Teach Us about Humanity. New Haven, CT: Yale University Press.

Bradshaw, G. A., Theodora Capaldo, Lorin Lindner, and Gloria Grow. 2008. "Building an Inner Sanctuary: Complex PTSD in Chimpanzees.” Journal of Trauma \& Dissociation 9(1): 934.

Bradshaw, G. A., Joseph P. Yenkowsky, and Eileen McCarthy. 2009. “Avian Affective Dysregulation: Psychiatric Models and Treatment for Parrots in Captivity." Proceedings of 30th Annual Association of Avian Veterinaries Conference.

Brewin, Chris R. 2001. "Memory Processes in Post-Traumatic Stress Disorder.” International Review of Psychiatry 13:159-63.

Bueter, Anke. 2019. "A Multi-Dimensional Pluralist Response to the DSM-controversies.” Perspectives in Science 27:316-43. 
Byrne, R. W., L. A. Bates, and C. J. Moss. 2009. "Elephant Cognition in Primate Perspective.” Comparative Cognition and Behavior Reviews 4:65-79.

Capaldo, Theodora, and G. A. Bradshaw. 2011. "The Bioethics of Great Ape Well-Being." Animals and Society Institute.

Carruthers, Peter. 1998. "Animal Subjectivity." Psyche 4(3).

Chomsky, N. 1959. "Review of Verbal Behavior.” Language 35:26-58.

Cooke, R. F., et al. 2017. "Effects of a Simulated Wolf Encounter on Brain and Blood Biomarkers of Stress-Related Psychological Disorders in Beef Cows with or without Previous Exposure to Wolves.” Journal of Animal Science 95(3): 1154-63.

Cooper, Rachel. 2006. “What Is Wrong with the DSM?” History of Psychiatry 15:5-25.

Dalgleish, T. 2004. “Cognitive Approaches to Posttraumatic Stress Disorder: The Evolution of Multirepresentational Theorizing." Psychological Bulletin 130:228-60.

Diagnostic and Statistical Manual of Mental Disorders (DSM-V). American Psychiatric Association. 2013. https://www.psychiatry.org/psychiatrists/practice/dsm.

Dodman, Nicholas H. 2016. Pets on the Couch: Neurotic Dogs, Compulsive Cats, Anxious Birds, and the New Science of Animal Psychiatry. New York: Atria.

Drell, M. J., C. H. Siegel, and T. J. Gaensbauer. 1993. “Post-traumatic Stress Disorder.” In Handbook of Infant Mental Health, edited by C. H. Zeanah. New York: Guilford Press.

Feldman, Ruth, and Adva Vengraber. 2011. "Posttraumatic Stress Disorder in Infants and Young Children Exposed to War-Related Trauma." Journal of the American Academy of Child Adolescent Psychiatry 50(7): 645-58. 
Ferdowsian, Hope R., et al. 2011. "Signs of Mood and Anxiety Disorders in Chimpanzees." PLOS ONE 6(6).

Fodor, J. 1968. Psychological Explanation. New York: Random House.

Ford, Julian D., et al. 2015. "Etiology of PTSD: What Causes PTSD?” In Posttraumtic Stress Disorder, 2nd ed., 81-112. Elsevier Inc.

Graf, Anna, Clemens Schiesti, and Markus A. Landolt. 2011. "Posttraumatic Stress and Behavior Problems in Infants and Toddlers with Burns." Journal of Pediatric Psychology 36(8): $923-31$.

Hempel, C. 1966. “The Logical Analysis of Psychology.” In Readings in Philosophical Analysis, edited by H. Feigl and W. Sellars. Englewood Cliffs, NJ: Prentice-Hall.

Hull, C. L. 1943. Principles of Behavior: An Introduction to Behavior Theory. New York: Appleton-Century.

Inoue, Sana, and Tetsuro Matsuzawa. 2007. "Working Memory of Numerals in Chimpanzees." Current Biology 17(23): R1004-R1005.

Kennedy, J. S. 1992. The New Anthropomorphism. London: Cambridge University Press.

King, Robert. 2010. “Complex Post-Traumatic Stress Disorder: Implications for Individuals with Autism Spectrum Disorders_-Part I.” Journal on Developmental Disabilities 16(3): 91100.

Lalumera, Elisabetta. 2016. "Saving the DSM-5? Descriptive Conceptions and Theoretical Concepts of Mental Disorders." Medicia E Storia 9-10:109-29. 
Levin, Janet. 2018. "Functionalism." The Stanford Encyclopedia of Philosophy (Fall edition), Edward N. Zalta (ed.), URL =https://plato. stanford.edu/archives/fall2018/entries/ functionalism/.

Mehtar, Mohamad, and Nahit Motavali Mukaddes. 2011. "Posttraumatic Stress Disorder in Individuals with Diagnosis of Autistic Spectrum Disorders." Research in Autism Spectrum Disorders 5(1): 539-46.

Nagasawa, Miho, Kazutaka Mogi, and Takefumi Kikusui. 2012. "Continued Distress among Abandoned Dogs in Fukushima." Scientific Report 2.

Putnam, Hilary. 1975. Mind, Language and Reality: Philosophical Papers, vol. 2. Cambridge: Cambridge University Press.

Ratcliffe, Matthew. 2015. Experiences of Depression: A Study in Phenomenology. Oxford: Oxford University Press.

Ratcliffe, Matthew. 2013. "The World of Depression.” Unpublished paper, available at academic.edu.

Revkin, Andrew C. August 10, 1995. “Chimp Research Laboratory Is Taken Over by Foundation." New York Times.

Scheeringa, Michael, et al. 2001. "Toward Establishing Procedural, Criterion, and Discriminant Validity for PTSD in Early Childhood.” Journal of the American Academy of Child Adolescent Psychology 40(1): 52-60.

Scheeringa, Michael, et al. 1995. "Two Approaches to the Diagnosis of Posttraumatic Stress Disorder in Infancy and Early Childhood." Journal of the American Academy of Child Adolescent Psychology 34(2): 191-200. 
Serova, L. I., A. Tillinger, M. Alaluf, et al. 2013. "Single Intranasal Neuropeptide Y Infusion Attenuates Development of PTSD-like Symptoms to Traumatic Stress in Rats.” Neuroscience 236:298-312.

Sheldrick, Dame Daphne. 2012. Love, Life, and Elephants: An African Love Story. New York: Farrar, Straus and Giroux.

Siebert, Charles. 2016. "What Does a Parrot Know about PTSD?” New York Times, 20 January. Skinner, B. F. 1953. Science and Human Behavior. New York: Macmillan.

Skinner, B. F. 1938. The Behavior of Organisms: An Experimental Analysis. New York: Appleton-Century.

Skinner, B. F. 1948. Walden Two. New York: Macmillian.

Stone, A., J. Turkkan, C. Bachrach, et al. (eds). 2009. The Science of Self-Report: Implications for Research and Practice. Mahwah: Lawrence Erlbaum Associates.

Tekin, Şerife, and Melissa Mosko. 2016. "Hyponarrativity and Context-Specific Limitations of the DSM-5.” Public Affairs Quarterly 29:109-29.

Thorndike, E. L. 1905. The Elements of Psychology. New York: A. G. Seiler.

Tolman, E. C. 1948. “Cognitive Maps in Rats and Men.” Psychological Review 55(4): 189-208.

Tsou, Jonathan Y. 2016. "Natural Kinds, Psychiatric Classification and the History of the DSM." History of Psychiatry 27:406-24.

Vermeire, S., K. Audenaert, A. Dobbeleir, et al. 2009. "Regional Cerebral Blood Flow Changes in Dogs with Anxiety Disorders Measured with SPECT.” Brain Imaging and Behavior $3: 342$.

Watson, J. 1913. "Psychology as the Behaviorist Views It.” Psychological Review 20:158-77. 
Wilson, John P. 2007. “The Lens of Culture: Theoretical and Conceptual Perspectives.” In Cross-Cultural Assessment of Psychological Trauma and PTSD, edited by John P.

Wilson and Catherine So-kum Tang, 3-30. Boston: Springer.

Zanette, Liana et al. 2019. "Predator-Induced Fear Causes PTSD-like Changes in the Brains and Behaviour of Wild Animals." Scientific Reports 9(1): 1-10. 